\title{
Opioid-Induced Bowel Dysfunction in Patients Undergoing Spine Surgery: Comparison of Oxycodone and Oxycodone-Naloxone Treatment
}

\author{
Merja Kokki · Moona Kuronen · Toivo Naaranlahti · Timo Nyyssönen • \\ Ira Pikkarainen · Sakari Savolainen · Hannu Kokki
}

Received: October 27, 2016/Published online: December 5, 2016

(C) The Author(s) 2016. This article is published with open access at Springerlink.com

\begin{abstract}
Introduction: Opioids are needed for postoperative pain in spine surgery patients, but opioid-induced constipation is a harmful adverse event. The aim of this clinical trial was to compare the use of a controlled-release oxycodone-naloxone combination product with oxycodone controlled-release tablets in
\end{abstract}

Enhanced content To view enhanced content for this article go to http://www.medengine.com/Redeem/ C037F060697B3BB9.

M. Kokki and M. Kuronen contributed equally.

M. Kokki $(\bowtie) \cdot$ M. Kuronen · I. Pikkarainen .

H. Kokki

Department of Anaesthesia and Operative Services,

Kuopio University Hospital, Kuopio, Finland

e-mail: hannu.kokki@uef.fi

M. Kokki · M. Kuronen · I. Pikkarainen · H. Kokki Faculty of Health Sciences, School of Medicine, University of Eastern Finland, Kuopio, Finland

T. Naaranlahti

Hospital Pharmacy, Kuopio University Hospital, Kuopio, Finland

\section{T. Nyyssönen}

Department of Orthopaedics, Kuopio University

Hospital, Kuopio, Finland

S. Savolainen

Department of Neurosurgery, Neuro Centre, Kuopio

University Hospital, Kuopio, Finland these patients. The main outcome measure was the prevalence of constipation at 7 days postoperatively assessed with a Bowel Function Index questionnaire. A follow-up assessment at 21 days after surgery was also included.

Methods: A total of 180 patients undergoing spine surgery, 91 having preoperative opioids in use and 89 opioid-naïve, were randomized to receive twice-daily oxycodone $10 \mathrm{mg}$ or oxycodone-naloxone 10/5 mg controlled-release tablets for the first 7 postoperative days. Patients were followed-up for 21 days after surgery.

Results: At baseline, prevalence of constipation was common both in the opioid-naïve-25/87 (29\%) and on-opioid groups 43/90 (48\%) $(P=0.009)$. This increased at 7 days postoperatively with no difference between the groups, 54/89 with oxycodone and 54/88 with oxycodone-naloxone had constipation. At 21 days, constipation was less than in the baseline in both groups, in the opioid-naive group the prevalence of constipation was 3/43 (7\%) in patients with oxycodone-naloxone compared to $9 / 44$ (21\%) with oxycodone (effect size 0.68; $P=0.068$ ). Both study compounds provided similar pain relief and were well tolerated. 
Conclusion: In patients presented for back surgery, the prevalence of constipation was significantly higher than that in the community. In opioid-naïve subjects, oxycodone-naloxone was beneficial concerning constipation; but this was not distinguishable in subjects with chronic opioid use. The analgesic efficacy of oxycodone and oxycodone-naloxone was similar.

Trial registration: European Clinical Trials Database (EudraCT no. 2012-001816-42) and ClinicalTrials.gov database (Identifier: NCT02573922).

Keywords: Constipation; Opioid induced bowel dysfunction (OIC); Oxycodone; Oxycodone-naloxone; Spine surgery

\section{INTRODUCTION}

Spine surgery is associated with significant postoperative pain and most patients need opioid analgesics during the early recovery period [1]. Although opioids are highly effective analgesics, they are associated with several adverse effects, with dizziness, somnolence, nausea, vomiting and constipation being the most common [2]. Some of these adverse effects are relieved within the first days of use, but constipation remains constant [3]. Opioid-induced constipation (OIC) is caused by the opioid agonist binding to the opioid peptide receptors distributed in the gastrointestinal canal. For many patients, OIC is harmful and may even delay discharge after surgery. [4-6].

In many countries, the use of oxycodone has surpassed that of morphine [7], and in Finland oxycodone has been the most commonly used opioid analgesic for postoperative pain for decades [8]. A few years ago, a prolonged-release oxycodone-naloxone combination product was approved for management of severe pain, which can be adequately managed only with opioid analgesics. In the combination product, the opioid antagonist naloxone is added to counteract OIC by blocking the action of oxycodone at the opioid receptors locally in the gut [9]. The systemic bioavailability of naloxone by mouth is relatively small, less than $3 \%$ [10], and it allows selective blocking of intestinal opioid receptors without decreasing the central nervous system analgesic efficacy of oxycodone as long as first-pass metabolism in liver is not surpassed.

Controlled-release oxycodone formulations provide relatively constant plasma concentrations [11] and seem to be feasible options in the treatment of postoperative pain [12, 13]. Oxycodone-naloxone controlled-release tablets are increasingly used for postoperative pain in order to decrease the risk of OIC [14], and preliminary experience suggests that this combination may also decrease other opioid-induced adverse events, like urinary retention [13]. Long-term efficacy and safety of oxycodone-naloxone has been demonstrated in palliative care, indicating that the combination product results in sustained analgesia, improved bowel function and improved symptoms of constipation [15].

In the present study, our hypothesis was that the oxycodone-naloxone combination product may decrease the incidence of OIC but preserve the analgesic efficacy compared to that of oxycodone without naloxone. We tested this hypothesis in two patient groups having elective spine surgery, on opioid-naïve patients and on those with chronic opioid treatment on arrival at the surgery. 


\section{METHODS}

The study was a prospective, randomized, single-blind parallel group and active-controlled study in two cohorts of patients who came for elective spine surgery to be performed in Kuopio University Central Hospital between 21 October 2012 and 21 April 2013. The study design was approved by the Research Ethics Committee of the Hospital District of Northern Savo, Kuopio, Finland (no. 41/2012), and was conducted in accordance with the Helsinki Declaration. The National Agency for Medicines was notified (no. 60/2012), and the study was recorded in the European Clinical Trials Database (EudraCT no. 2012-001816-42) and in the ClinicalTrials.gov database (Identifier: NCT02573922). The study had institutional approval.

A flow chart is presented in Fig. 1. A total of 180 patients gave their informed consent for participation in the trial. The study had two arms: subjects who had continuous opioid use before surgery $(n=91)$ and opioid-naïve subjects $(n=89)$. These two cohorts were randomly assigned to receive either oxycodone controlled-release $10 \mathrm{mg}$ tablets (Oxycontin ${ }^{\circledR}$; Mundipharma, Vantaa, Finland) or oxycodone-naloxone controlled-release 10/5 mg tablets (Targiniq ${ }^{\circledR}$; Mundipharma) in the morning and evening as long as their postoperative pain was moderate to severe or for a maximum of 7 days, i.e. a total of 14 tablets. All medications were obtained from commercial sources. The randomization list was generated by computer (http://www. randomization.com), and a sealed envelope method was used for blinding on enrolment. A hospital ward pharmacist prepared and concealed the random assignments from other persons involved in the study.
We recruited male and female patients aged 18-75 years who were scheduled to have an elective lumbar or cervical spinal surgery, who had a body mass index of $18-35 \mathrm{~kg} / \mathrm{m}^{2}$ and who had an American Society of Anesthesiologists physical status classification of I-III. At enrolment, we excluded patients with sleep apnoea or other diseases affecting the respiratory center, renal, hepatic or pulmonary impairment, history of alcohol or narcotic abuse, or an allergy to oxycodone, naloxone or excipients. We did not enrol women who were lactating or pregnant.

In the opioid-naïve group, subjects should not have been given any opioids in the 4 weeks prior to the study recruitment. Subjects on-opioids were switched from the opioid in use to the study compound in the morning before surgery and switched back to the original opioid after 7 days on the study compound, i.e. on the 7 th postoperative morning.

A standardized endotracheal anesthesia was used in all subjects. The subjects received diazepam $10 \mathrm{mg}$ and paracetamol $2 \mathrm{~g}$ by mouth as premedication. Anesthesia was induced with midazolam 1-2.5 mg and propofol i.v., remifentanil-infusion was used for intraoperative analgesia and rocuronium to facilitate tracheal intubation. Anesthesia was maintained with desflurane in oxygen in air, and desflurane inhalation was adjusted to maintain the response and state entropy values between 40 and 60 (Carescape ${ }^{\mathrm{TM}}$ B650; GE Healthcare, Helsinki, Finland). At the end of anesthesia, sugammadex was used for reversal of muscle relaxation.

For postoperative pain management in hospital, subjects were prescribed paracetamol $1 \mathrm{~g}$ i.v. and then three times a day by mouth, and dexketoprofen, a non-steroidal anti-inflammatory analgesic (NSAID), 


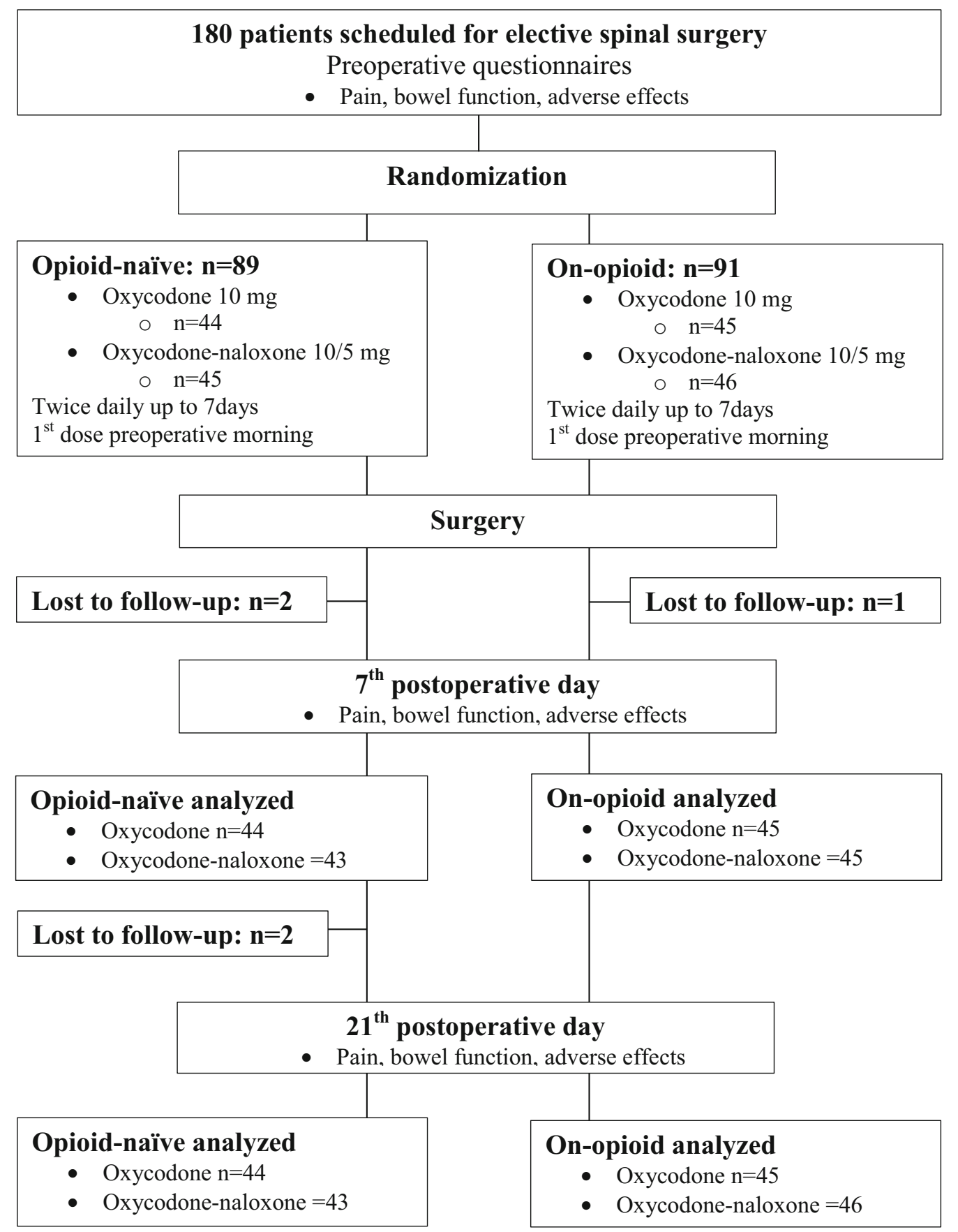

Fig. 1 Flow chart

25-50 mg i.v., followed by meloxicam $7.5 \mathrm{mg}$ by mouth twice a day. For rescue analgesia in hospital, subjects had oxycodone $2-3 \mathrm{mg}$ i.v./ immediate release $5-10 \mathrm{mg}$ capsules by mouth/ s.c. injection.
At discharge subjects were given the rest of the 14 tablet blister of the study medication from the hospital with appropriate counseling and labeling, and were asked to return the unused tablets in the follow-up visit. Other 
postoperative pain treatment was prescribed according to the medical judgement of the attending physician; most were prescribed paracetamol and/or NSAID (meloxicam/ ibuprofen), 18 had pregabalin, 2 gabapentin and 2 tizanidine as a concomitant medication. After 7 days, if needed, the subjects on-opioid were switched back to the initial opioid that had been in use before surgery.

The subjects were asked to assess their bowel function and pain scores at baseline before surgery, at discharge, and at 1 and 3 weeks after surgery. The questionnaires at baseline and at discharge were filled out by the subjects themselves and checked by a study nurse to ensure the completeness of the data. After discharge, the subjects were phone-interviewed at 7 and 21 days after surgery using the same structured questionnaires as at baseline.

Bowel function was assessed using the Bowel Function Index (BFI) questionnaire [16]. The BFI is calculated as a mean of three variables, ease of defecation, feeling of incomplete bowel evacuation and personal judgement of constipation on a numerical rating scale (NRS) 0-100. We used here a modified 11-point NRS $(0=$ no symptom, $10=$ most severe symptom $)$. The cut-off value for constipation was set as $3 / 10$ or higher.

Pain severity and pain relief provided by analgesics was evaluated on an 11-point NRS $(0=$ no $\quad$ pain $/$ no pain relief, $10=$ most pain/complete pain relief). The use of analgesics, laxatives, dietary changes to relief constipation and all adverse events were asked and recorded for each subject daily during the hospitalization, at discharge, and at 7 days and 21 days after surgery.

The primary outcome variable was the prevalence of constipation at 7 days after surgery. Secondary outcome variables were the prevalence of constipation at 21 days after surgery, and the pain intensity and the pain relief achieved with the study compound at 7 days after surgery. Safety was assessed by monitoring subjects for suspected adverse drug events (ADEs).

\section{Statistics}

The sample size calculation was based on the incidence of constipation in patients with chronic opioid use of $41 \%$ [2]. In order to decrease the incidence of constipation from 41 to $14 \%$, i.e. that in the community [17], with $80 \%$ power using a significance level of 0.05 , a minimum of 42 subjects per group was needed. In case of drop-outs, 45 subjects per group and a total of 180 subjects were recruited.

The data were entered and analyzed using IBM SPSS Statistics for Windows, v.23.0 (IBM, Armonk, NY, USA). Data are presented as median (minimum and maximum), mean (standard deviation, SD) or number of cases, as appropriate. For the primary outcome variable, we calculated 95\% confidence intervals (CI). Continuous data were tested with the Mann-Whitney $U$ test, and $\chi^{2}$ test and McNemar test were used for comparing proportions, as appropriate. All statistical tests were performed on an exploratory basis with a two-sided level of significance. A $P$ value of $<0.05$ indicated statistical significance.

\section{RESULTS}

The patient characteristics are presented in Table 1. There were no significant differences in the demographic variables between the four groups. In the on-opioid group, four subjects with codeine also used another opioid, tramadol $(n=3)$ and buprenorphine $(n=1)$, and one subject used both oxycodone and morphine. 
Table 1 Patient characteristics

\begin{tabular}{|c|c|c|c|c|}
\hline \multirow[t]{2}{*}{ Parameter } & \multicolumn{2}{|c|}{ Opioid naïve $(n=89)$} & \multicolumn{2}{|c|}{ On-opioid $(n=91)$} \\
\hline & $\begin{array}{l}\text { Oxycodone } \\
(n=44)\end{array}$ & $\begin{array}{l}\text { Oxycodone-naloxone } \\
(n=45)\end{array}$ & $\begin{array}{l}\text { Oxycodone } \\
(n=45)\end{array}$ & $\begin{array}{l}\text { Oxycodone-naloxone } \\
(n=46)\end{array}$ \\
\hline Age (years) & $51(25-67)$ & $54(40-75)$ & $51(20-73)$ & $53(23-71)$ \\
\hline Sex (female/male) & $21 / 23$ & $18 / 27$ & $24 / 21$ & $18 / 28$ \\
\hline Height $(\mathrm{cm})$ & $1.70(1.55-1.92)$ & $1.71(1.54-1.87)$ & $1.71(1.54-1.92)$ & $1.75(1.58-1.88)$ \\
\hline Weight (kg) & $76(50-110)$ & $81(53-113)$ & $80(52-115)$ & $81(55-110)$ \\
\hline BMI $\left(\mathrm{kg} / \mathrm{m}^{2}\right)$ & $26.1(20.8-31.8)$ & $26.6(18.8-35.4)$ & $26.7(20.3-34.6)$ & $27.1(20.0-35.1)$ \\
\hline \multicolumn{5}{|l|}{ Opioid in use } \\
\hline Codeine & & & 17 & 29 \\
\hline Tramadol & & & 16 & 13 \\
\hline Buprenorphine & & & 5 & 1 \\
\hline Oxycodone & & & 7 & 3 \\
\hline \multicolumn{5}{|l|}{ Type of surgery } \\
\hline Cervical/thoracic/lumbar spine & $27 /-/ 17$ & $21 / 1 / 23$ & $26 /-/ 19$ & $28 / 2 / 16$ \\
\hline
\end{tabular}

Data are median (minimum-maximum) or number of patients $B M I$ body mass index

\section{Baseline}

At baseline, the prevalence of constipation (BFI $\geq 3 / 10$ ) was high in both groups and it was higher in the on-opioid group, 43/91, compared to the opioid-naïve group, $25 / 89$, respectively $(P=0.009)$.

The subjects in the on-opioid group reported more pain at baseline compared to subjects in the opioid-naïve group. Pain during the previous $24 \mathrm{~h}$ was as followed: an average median 5 (range, 0-9) versus 4 (0-9) $(P=0.011) ;$ most $7 \quad(2-10)$ versus 7 (1-9) $(P=0.016)$ and right now $5(0-9)$ versus 4 (0-9) $(P=0.025)$, in the on-opioid group and the opioid-naïve group, respectively. The least pain was similar, $3(0-7)$ versus $2(0-7)$ $(P=0.68)$ in the on-opioid group and the opioid-naïve group, respectively.
At baseline opioid analgesics were reported to provide better pain relief than non-opioid analgesics: the median score of pain relief in the on-opioid group was $6(0-10)$ compared to 5 $(0-10) \quad(P=0.001)$ in the opioid-naïve group (Tables 2, 3).

\section{Opioid-Naïve Group}

Adherence to study medication was high, with most subjects, 67/89, using all the 14 study tablets. In the oxycodone group, 9 subjects, and in the oxycodone-naloxone group, 13 subjects, ceased the study medication before 7 days. Three in the oxycodone group and eight in the oxycodone-naloxone group did not use all tablets as they had only mild pain or no pain; three in each group due to suspected ADEs and 


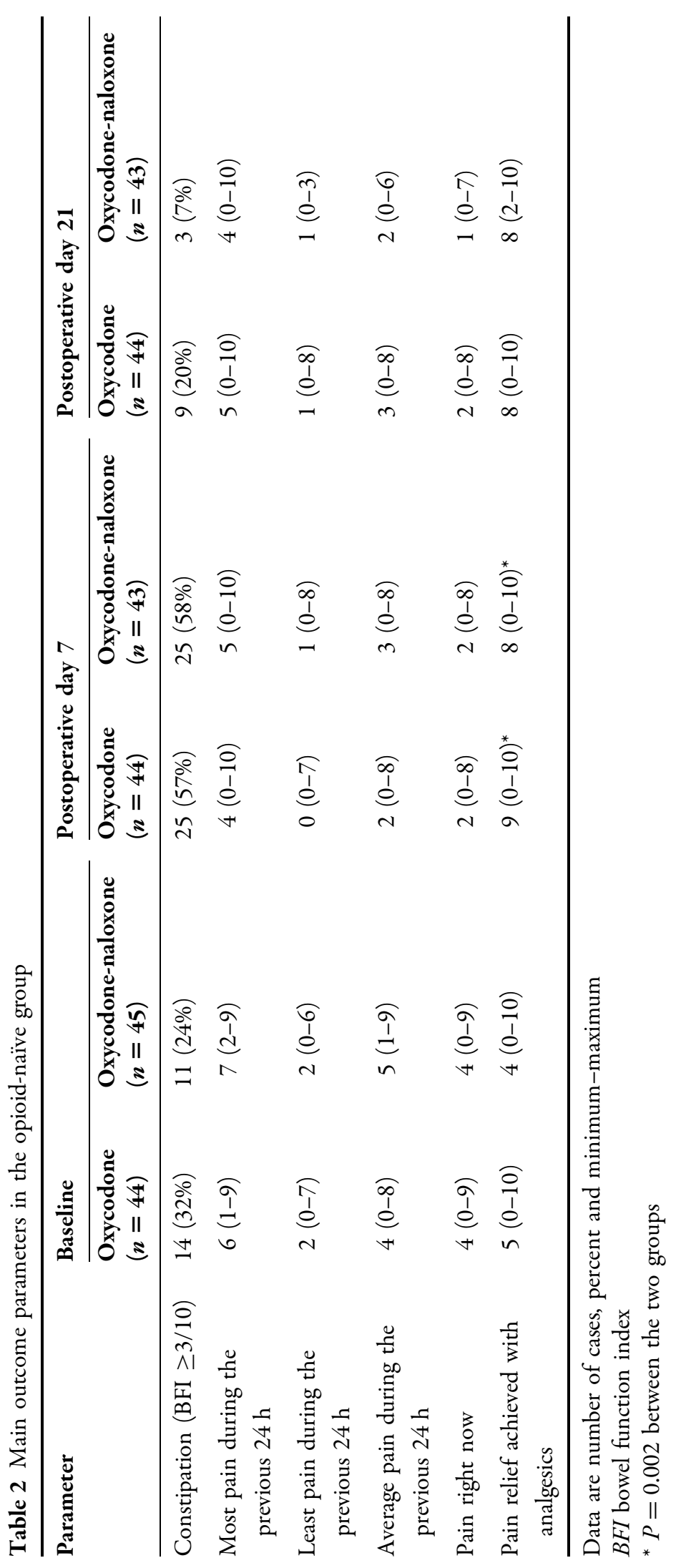




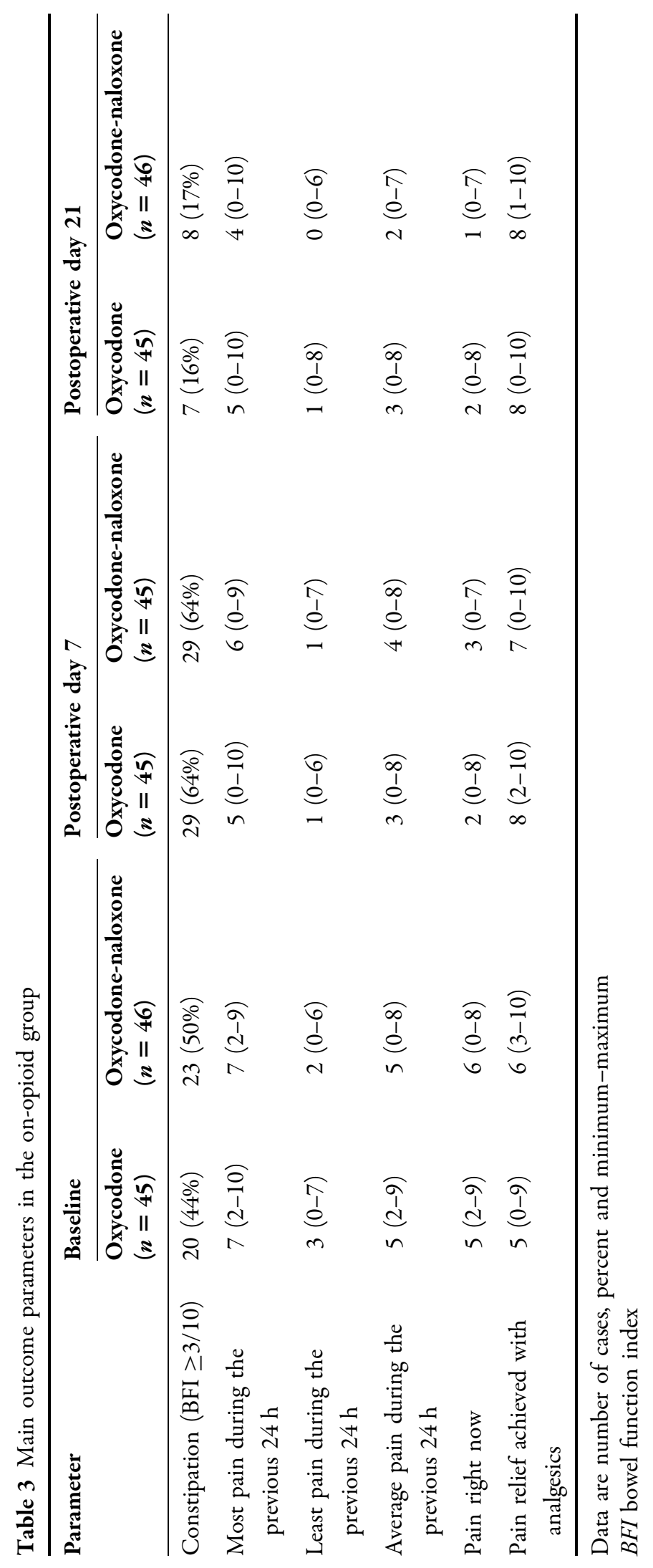


two subjects in each group did not give any specific reason.

At baseline BFI was similar in both groups: in the oxycodone group median $1.0(0.0-7.7)$ and in the oxycodone-naloxone group $1.2(0.0-5.7)$.

In the opioid-naïve group, the prevalence of constipation increased compared to baseline in both groups at 7 days after surgery: in the oxycodone group from 32 to 57\% (95\% CI for difference, 12-38) and in the oxycodone-naloxone group from 24 to 58\% (95\% CI 20-48). However, at 21 days after surgery, i.e. 2 weeks after opioid administration, the prevalence of constipation was less than that at baseline $(P=0.007)$ : in the oxycodone group 20\% (95\% CI for difference compared to baseline, $2-21)(P=0.18)$ and in the oxycodone-naloxone group 7\% (95\% CI 6-29) $\quad(P=0.039$ compared to baseline; $P=0.068$ between the two groups) (Table 2 ).

The use of laxatives ( $n=11$ vs. 9), dietary changes (increased intake of fibre/drinking plenty of water) ( $n=12$ vs. 8$)$ or use of enemas ( $n=4$ vs. 1$)$ were more common in the oxycodone group, 25 out of 44 subjects, than in the oxycodone-naloxone group, 15 out of 43 subjects $(P=0.04)$, respectively.

At 3 weeks after surgery, BFI was significantly lower than that at baseline in both groups: in the oxycodone group median $0.0(0.0-6.0)$ and in the oxycodone-naloxone group $0.0(0.0-3.7)$ $(P<0.001)$. Nine subjects $(21 \%)$ in the oxycodone group and three (7\%) in the oxycodone-naloxone group had BFI $\geq 3.0$ $(P=0.068)$. In the oxycodone group, 7 of the 14 who did have constipation at baseline had constipation at 21 days compared to 1 of the 11 in the oxycodone-naloxone group $(P=0.028)$ (Table 2).

There was no difference between the two groups in the pain intensity at 7 and 21 days after surgery. Both study compounds were reported to provide high analgesic efficacy: at 7 days after surgery in the oxycodone group the median of pain relief was $9(0-10)$ and that in the oxycodone-naloxone group 8 (0-10) $(P=0.002)$.

Adverse drug events were common in both groups: 30 out of 44 subjects in the oxycodone group reported a total of 51 suspected ADEs during the first 21 days after surgery compared with 23 out of 45 subjects with 32 ADEs in the oxycodone-naloxone group. The incidence of suspected ADEs was 17\% (95\% CI 9-25) higher in the oxycodone group than in the oxycodone-naloxone group $(P=0.13)$. However, neither serious nor unexpected ADEs were reported. One subject had a wound infection (Table 4).

\section{On-Opioid group}

In the on-opioid group, the need for opioid analgesia was higher than in the opioid-naïve group, with all except five subjects in both groups using all the study tablets. One subject in the oxycodone group had only mild pain and two subjects ceased the study medication due to suspected ADEs. In the oxycodone-naloxone group, three subjects ceased the study medication due to suspected ADEs. Two subjects in both groups did not give any specific reason for the early cessation.

At baseline, BFI was similar in both groups: in the oxycodone group $2.3(0.0-9.0)$ and in the oxycodone naloxone group 3.3 (0.0-9.0). Also, the proportion of patients with $\mathrm{BFI} \geq 3.0$ was similar in the two groups: $20 / 45(44 \%)$ in the oxycodone group and $23 / 46(50 \%)$ in the oxycodone-naloxone group.

At 7 days after surgery, the prevalence of constipation increased non-significantly in both groups. At 21 days after surgery, i.e. 2 weeks after the study compound 
Table 4 Suspected adverse drug events (ADE)

\begin{tabular}{|c|c|c|c|c|}
\hline \multirow[t]{2}{*}{ Parameter } & \multicolumn{2}{|c|}{ Opioid-naïve $(n=89)$} & \multicolumn{2}{|c|}{ On-opioid $(n=91)$} \\
\hline & $\begin{array}{l}\overline{\text { Oxycodone }} \\
(n=44)\end{array}$ & $\begin{array}{l}\text { Oxycodone-naloxone } \\
(n=45)\end{array}$ & $\begin{array}{l}\text { Oxycodone } \\
(n=45)\end{array}$ & $\begin{array}{l}\text { Oxycodone-naloxone } \\
(n=46)\end{array}$ \\
\hline Subjects with adverse events & $30(68 \%)$ & $23(51 \%)$ & $34(73 \%)$ & $32(70 \%)$ \\
\hline Total number of ADEs & 51 & 32 & 60 & 49 \\
\hline Constipation & 14 & 7 & 20 & 17 \\
\hline Somnolence & 4 & 4 & 7 & 7 \\
\hline Nausea & 4 & 7 & 5 & 4 \\
\hline Dizziness & 4 & 2 & 5 & 5 \\
\hline Abdominal irritation & 6 & 3 & 4 & 1 \\
\hline Dry mouth & 3 & - & 2 & 5 \\
\hline Abdominal pain & 2 & 2 & 2 & 1 \\
\hline Confusion & 2 & - & 2 & 2 \\
\hline Sleeping disturbed & 2 & 2 & - & 2 \\
\hline Diarrhoea & 1 & 1 & 1 & 1 \\
\hline Voiding difficulty & 2 & - & 1 & 1 \\
\hline Eye irritation/visual symptoms & 2 & - & 1 & - \\
\hline Flatulence & 1 & 2 & - & - \\
\hline Headache & 2 & - & 1 & - \\
\hline Heartburn & 1 & - & 1 & 1 \\
\hline Withdrawal symptoms & - & - & 2 & 1 \\
\hline Vomiting & 1 & - & 1 & - \\
\hline Sweating & - & 1 & 1 & - \\
\hline Anxiety & - & & 1 & \\
\hline Insufficient efficacy & 1 & - & - & - \\
\hline Mood changes & - & & 1 & \\
\hline Oedema & - & - & 1 & - \\
\hline Pruritus & 1 & - & - & - \\
\hline Shivering & - & - & 1 & - \\
\hline Shortness of breath & - & & - & 1 \\
\hline Tachycardia & - & 1 & - & - \\
\hline
\end{tabular}

Data are number of cases (\%) 
administration, the prevalence of constipation was less than that at baseline in both groups: in the oxycodone group $16 \% \quad(95 \%$ CI for difference compared to baseline, 17-42) and in the oxycodone-naloxone group $17 \%$ (95\% CI 19-46) of subjects had BFI $>3.0$. In the oxycodone group, five who did not have constipation at baseline had constipation at 21 days compared with one in the oxycodone-naloxone group $(P=0.081)$ (Table 3). In the on-opioid group, half of the subjects had interventions to treat constipation: 28 out of 44 subjects in the oxycodone group and 21 out of 46 in the oxycodone-naloxone group $(P=0.11)$. Laxatives were used by 20 subjects in the oxycodone group compared to 13 in the oxycodone-naloxone group, and dietary changes by 10 compared to 11 and enema by 1 compared to 2 subjects, respectively. One subject in the oxycodone group needed a manual extraction.

At 3 weeks after surgery, BFI was lower than that in the baseline in both groups: the mean decrease in the oxycodone-naloxone group, 2.0 (95\% CI 1.3-2.8) $(P<0.001)$, and in the oxycodone group, $1.6 \quad(95 \%$ CI $\quad 0.7-2.6)$ $(P=0.003)$.

There was no difference between the two groups in the pain intensity and pain relief achieved with the study compound at 7 and 21 days after surgery. Both groups reported high analgesic efficacy with oxycodone: at 7 days after surgery in the oxycodone group, the median of pain relief was $8(2-10)$, and in the oxycodone-naloxone group $7(0-10)(P=\mathrm{NS})$.

Adverse drug events were more common in the on-opioid group than in the opioid-naïve group $(P=0.066)$, but neither serious nor unexpected ADEs were reported. A total of 34 out of 45 subjects in the oxycodone group reported a total of 60 ADEs during the first 21 days after surgery compared to 32 out of 46 subjects with 49 ADEs in the oxycodone-naloxone group $(P=0.52)$. One subject had a wound infection (Table 4 ).

\section{DISCUSSION}

A novel finding in the present study was the high prevalence of constipation at baseline both in the opioid-naïve subjects, 29\%, and in subjects having chronic opioid use before surgery, $48 \%$. These numbers are several-fold higher than the prevalence of idiopathic constipation in the community, $4 \%$ in men and $8 \%$ in women in Finland [18] and 14\% in North America [17], but the prevalence in the on-opioid group was similar to that reported by Kalso and coworkers [2] in patients with chronic opioid use. Factors increasing intrathoracic or intra-abdominal pressures, such as defecation, are known to raise epidural space pressure and, as a consequence, may enhance symptoms in patients with back diseases [19]. This may lead to a fear of defecation and result in constipation. Thus, it is important to take bowel function into account while planning the treatment path in patients scheduled for back surgery.

Another novel finding was the efficiency of the combination product on the severity of constipation. Our study hypothesis was that the oxycodone-naloxone combination product would result in a lower prevalence of OIC than oxycodone without naloxone, but that a small amount of naloxone by mouth, $10 \mathrm{mg}$ daily, might not decrease the analgesic efficacy of oxycodone. In the early recovery phase after surgery, almost two-thirds of the subjects had constipation assessed by BFI and there was no difference between the subjects who had oxycodone or oxycodone-naloxone. However, as an $\mathrm{ADE}$, constipation was reported two times less commonly in subjects with 
oxycodone-naloxone among opioid-naïve subjects, and the need for interventions to release constipation was less in the oxycodone-naloxone group (36/89) than in subjects with oxycodone controlled-release tablets $(53 / 89, \quad P=0.009)$. Thus, opioid antagonists by mouth are increasingly being used to prevent and treat opioid-induced bowel dysfunction.

A further new finding was the observation that in the opioid-naïve group constipation was still relatively common at 2 weeks after opioid administration, but in the oxycodone-naloxone group the prevalence was similar to that in the community. The positive carry-on-effect of naloxone added to oxycodone found here is consistent with an earlier report indicating that some of the positive effects of naloxone may be evident even at 2 weeks after the use of the combination product [13].

Our results are consistent with earlier publications indicating that opioid-induced bowel dysfunction is a common problem in patients with chronic opioid use [2]. In some cases, constipation and associated symptoms can be so difficult that the patients will prefer to decrease or stop opioid medication and tolerate pain rather than stay on opioid analgesics [20]. Postoperative OIC is also frequent in surgical patients, with anesthesia, surgery, bed rest and use of perioperative opioids being the main causes of constipation in these patients. After major thoracolumbar fusion spinal surgery, almost half of the patients, $44 \%$, have severe constipation [21]. After orthopaedic surgery with postoperative opioid pain therapy, constipation was associated with a hospital stay prolonged by 1.4 days [6]. In another study, opioid-related ADEs including constipation after orthopedic surgery were associated with both hospital stay prolonged by 0.5 days and increased hospital costs [12].
In the present study, some benefits among on-opioid subjects were noticed by combining naloxone with oxycodone. In the oxycodone group, five who did not have constipation at baseline had constipation at 21 days compared with one in the oxycodone-naloxone group. Moreover, in the on-opioid study arm, both laxatives and other interventions to treat constipation were used more commonly in the oxycodone group than in the oxycodone-naloxone group. One subject in the oxycodone group even needed a manual extraction to relieve constipation. This increased use of interventions to relieve constipation is assumed to be the reason why the benefits of naloxone were not that evident in the on-opioid group as they were in the opioid-naïve group.

There have been questions whether oxycodone-naloxone provides non-inferior pain relief compared to prolonged-release oxycodone. Our data here and earlier [13] indicate that oxycodone doses of $10-40 \mathrm{mg}$ with naloxone in a ratio of 2:1 two times a day provide similar pain relief to prolonged-release oxycodone at the same doses. In the present study, the analgesic efficacy was similar for both study compounds except at 7 days after surgery when the opioid-naïve subjects with oxycodone reported better pain relief. However, this difference was not considered clinically meaningful, as the median of pain relief on a NRS-scale of $0-10$ was high in both groups, 9/10 and 8/10, respectively. In our earlier study in orthopaedic arthroplasty surgery, controlled-release tablets of oxycodone-naloxone and oxycodone were equally effective in pain treatment [13]. Supporting non-inferiority, similar pain relief and no differences in bowel function have also been reported in laparoscopic hysterectomy by 
Comelon and coworkers [14]. In the approved summary of product characteristics, prolonged-release oxycodone-naloxone tablets are not recommended for pre-operative use or within the first $12-24 \mathrm{~h}$ postoperatively. In our study, the first dose of the study drug was given in the morning, a few hours before surgery. In the Comelon and coworkers [14] study, the patients also received the first dose of oxycodone-naloxone $1-2 \mathrm{~h}$ before surgery, indicating that preoperative administration can be used safely. However, we are unaware of any direct comparison of pre- and postoperative initiation of administration that may clarify the situation.

There is no consensus on when it is cost-effective to use the combination product, as in some trials no benefit has been found regarding OIC with oxycodone-naloxone controlled-released tablets. Possibly, a too short use of the oxycodone-naloxone compound in studies by Kuusniemi and coworkers [13] and Comelon and coworkers [14] may have been the reason for the similar incidence on OIC with oxycodone-naloxone as with oxycodone tablets. In these two previous studies, the combination product was only used for $72 \mathrm{~h} / 5-6$ tablets compared to 7 days/14 tablets in the present study and 14 days/28 tablets in our pilot study [13]. In the present study, the 7-day treatment with the combination product performed a little better in opioid-naïve subjects than in on-opioid subjects. Taken together, it seems that, for the prevention of OIC, an anticipated need for opioid analgesia of 7 days oxycodone-naloxone controlled-released tablets can be an appropriate choice, and, secondly, that in patients on-opioid and already having opioid-induced bowel dysfunction, oxycodone-naloxone prolonged-release tablets could be a feasible option. Moreover, some of the benefits may be evident only later, as was the case here and in our previous study [13].

Both products were well tolerated and neither serious nor unexpected ADEs were reported. Suspected ADEs were typical for opioids. In the whole cohort, 64 out of 89 subjects in the oxycodone group and 55 out of 91 in the oxycodone-naloxone group developed ADEs, and the total number of ADEs was higher in the oxycodone group, $n=111$, than in the oxycodone-naloxone group, $n=82$, indicating that there could be differences in the adverse event profiles between these two products.

The main limitation of this trial was that it was not double-blinded and this may have affected the study results. However, matching placebos were not available. In order to decrease bias, allocation concealment was used to avoid selection bias and subjects were not informed about their group assignment. In hospital, study compounds were given in a medicine cup and at discharge the blisters were repacked in identical paper packs. Secondly, a limitation was that other analgesics treatment was not standardized and because multimodal pain therapy was applied. The present study was performed in a single center where uniform institutional guidelines concerning multimodal postoperative pain treatment are used. Most subjects had, for example, NSAID as a concomitant medication with a similar frequency across the study groups. This is an important point as experimental data indicate that NSAIDs may antagonize the constipation effects of some opioid analgesics [22]. Secondly, gabapentinoids were used in 20 patients to continue patients' preoperative medications. There are recommendations that gabapentinoids should not be withdrawn just before surgery in order to avoid withdrawal symptoms $[23,24]$. Thirdly, the prevention and 
management of constipation was not standardized. However, these results may reflect appropriately the current clinical situation where patients use over-the-counter laxatives and other constipation medications. Opioid-induced constipation seems to be poorly controlled by over-the-counter laxatives [25] possibly due to the mechanism of constipation. Gastrointestinal opioid receptor blockade and specific gastrointestinal affecting opioid antagonists may be more effective in treating OIC [26].

We believe these results are soundly based and applicable to other institutions performing spine surgery. Oxycodone and naloxone both are well known and extensively evaluated compounds in cancer and chronic pain management. There is evolving evidence that the analgesic efficacy of the combination product of oxycodone and naloxone is not inferior to oxycodone alone. However, more studies are needed concerning OIC after surgery. In these studies, the sufficient treatment duration is essential as 2 or 3 days treatment seem not to be that effective in order to prevent OIC $[13,14]$, but 7 -day treatment may benefit opioid-naïve patients as shown in the present study.

\section{CONCLUSION}

As a conclusion, in opioid-naïve patients, postoperative oxycodone-naloxone was beneficial concerning OIC and the severity of its symptoms: BFI was significantly lower and the proportion of patients with OIC was less than in patients with oxycodone after spine surgery. Moreover, the oxycodone-naloxone combination product had a carry-on effect in opioid-naïve subjects. This was not that evident in patients with chronic opioid use although numeric values also support the use of the combination product in them. Both oxycodone and oxycodone-naloxone prolonged-release tablets were equally efficacious in postoperative pain treatment and both were well tolerated.

\section{ACKNOWLEDGEMENTS}

The study, article-processing charges and the open access fees were funded by a Finnish governmental VTR-fund from the Hospital District of Northern Savo, Kuopio, Finland. The study was not financially supported by any external sources. All named authors meet the International Committee of Medical Journal Editors (ICMJE) criteria for authorship for this manuscript, take responsibility for the integrity of the work as a whole, and have given final approval to the version to be published.

Disclosures. Merja Kokki, Moona Kuronen, Toivo Naaranlahti, Timo Nyyssönen, Ira Pikkarainen, Sakari Savolainen and Hannu Kokki have nothing to disclose.

Compliance with Ethics Guidelines. All procedures were in accordance with the ethical standards of the Helsinki Declaration of 1964, as revised in 2013. The study design was approved by the Research Ethics Committee of the Hospital District of Northern Savo, Kuopio, Finland (no. 41/2012) and was conducted in accordance with the Helsinki Declaration. The National Agency for Medicines was notified (no. 60/2012) and the study was recorded in the European Clinical Trials Database (EudraCT no. 2012-001816-42) and in the ClinicalTrials.gov database (Identifier: NCT02573922). The study had an institutional approval. Written informed consent was obtained from each patient. 
Data Availability. The datasets during and/ or analyzed during the current study are available from the corresponding author on reasonable request.

Open Access. This article is distributed under the terms of the Creative Commons Attribution-NonCommercial 4.0 International License (http://creativecommons.org/licenses/ by-nc/4.0/), which permits any noncommercial use, distribution, and reproduction in any medium, provided you give appropriate credit to the original author(s) and the source, provide a link to the Creative Commons license, and indicate if changes were made.

\section{REFERENCES}

1. Gerbershagen H, Aduckathil S, van Wijck A, Peelen L, Kalkman C, Meissner W. Pain intensity on the first day after surgery: a prospective cohort study comparing 179 surgical procedures. Anesthesiology. 2013;118:934-44.

2. Kalso E, Edwards J, Moore R, McQuay H. Opioids in chronic non-cancer pain: systematic review of efficacy and safety. Pain. 2004;112:372-80.

3. Pappagallo M. Incidence, prevalence, and management of opioid bowel dysfunction. Am J Surg. 2001;182:11-8.

4. Portenoy R. Constipation in the cancer patient: causes and management. Med Clin N Am. 1987;71:303-11.

5. Bell T, Panchal S, Miaskowski C, Bolge S, Milanova $\mathrm{T}$, Williamson R. The prevalence, severity, and impact of opioid-induced bowel dysfunction: results of a US and European Patient Survey (PROBE 1). Pain Med. 2009;10:35-42.

6. Pizzi L, Toner R, Foley K, et al. Relationship between potential opioid-related adverse effects and hospital length of stay in patients receiving opioids after orthopedic surgery. Pharmacotherapy. 2012;32:502-14.

7. The Report of the International Narcotics Control Board for 2015 (E/INCB/2015/1), United Nations. https://www.incb.org/documents/Narcotic-Drugs/
Technical-Publications/2015/NAR-tech_pub_2015. pdf. Accessed 21 Oct 2016.

8. Hamunen K, Paakkari P, Kalso E. Trends in opioid consumption in the Nordic countries 2002-2006. Eur J Pain. 2009;13:954-62.

9. Kokki H, Kokki M, Sjövall S. Oxycodone for the treatment of post-operative pain. Expert Opin On Pharmacother. 2012;13:1045-58.

10. Smith K, Hopp M, Mundin G, et al. Low absolute bioavailability of oral naloxone in healthy subjects. Int J Clin Pharmacol Therap. 2012;50:360-7.

11. Kokki M, Välitalo P, Rasanen I, et al. Absorption of different oral dosage forms of oxycodone in the elderly: a cross-over clinical trial in patients undergoing cystoscopy. Eur J Clin Pharmacol. 2012;68:1357-63.

12. Rothwell M, Pearson D, Hunter J, et al. Oral oxycodone offers equivalent analgesia to intravenous patient-controlled analgesia after total hip replacement: a randomized, single-centre, non-blinded, non-inferiority study. Br J Anaesth. 2011;106:865-72.

13. Kuusniemi K, Zöllner J, Sjövall $\mathrm{S}$, et al. Prolonged-release oxycodone/naloxone in postoperative pain management: from a randomized clinical trial to usual clinical practice. J Int Med Res. 2012;40:1775-93.

14. Comelon M, Wisloeff-Aase K, Raeder J, et al. A comparison of oxycodone prolonged-release vs. oxycodone + naloxone prolonged-release after laparoscopic hysterectomy. Acta Anaesthesiol Scand. 2013;57:509-17.

15. Ahmedzai SH, Leppert W, Janecki $M$, et al. Long-term safety and efficacy of oxycodone/naloxone prolonged-release tablets in patients with moderate-to-severe chronic cancer pain. Support Care Cancer. 2015;23:823-30.

16. Rentz A, Yu R, Müller-Lissner S, Leyendecker P. Validation of the Bowel Function Index to detect clinically meaningful changes in opioid-induced constipation. J Med Econ. 2009;12:371-83.

17. Helakorpi S, Pajunen T, Jallinoja P, Virtanen S, Uutela A. Health behaviour and health among the Finnish adult population, Spring 2010. National Institute for Health and Welfare (THL), Report 15/2011, 203 pages. Helsinki 2011. ISBN 978-952-245-472-0 (print), ISBN 978-952-245-473-7 (pdf). https://www.thl.fi/ documents/10531/95613/Raportti\%202011\%2015. pdf. Accessed 15 Aug 2016. 
18. Suares N, Ford A. Prevalence of, and risk factors for, chronic idiopathic constipation in the community: systematic review and meta-analysis. Am J Gastroenterol. 2011;106:1582-91.

19. Usubiaga JE, Moya F, Usubiaga LE. Effect of thoracic and abdominal pressure changes on the epidural space pressure. Br J Anaesth. 1967;39:612-8.

20. Brock C, Olesen S, Olesen A, Frøkjaer JB, Andresen T, Drewes A. Opioid-induced bowel dysfunction: pathophysiology and management. Drugs. 2012;72:1847-65.

21. Stienen M, Smoll N, Hildebrandt G, Schaller K, Tessitore E. Gautschi. Constipation after thoraco-lumbar fusion surgery. Clin Neurol Neurosurg. 2014;126:137-42.

22. Planas E, Poveda R, Sánchez S, Romero A, Puig M. Non-steroidal anti-inflammatory drugs antagonise the constipating effects of tramadol. Eur J Pharmacol. 2003;482:223-6.

23. Summary of product characteristics Lyrica. http:// www.ema.europa.eu/docs/fi_FI/document_library/
EPAR_-_Product_Information/human/000546/WC 500046602.pdf. Accessed 15 Aug 2016.

24. Summary of product characteristics Neurontin. http://spc.fimea.fi/indox/nam/html/nam/humspc/ 7/10796177.pdf. Accessed 15 Aug 2016.

25. Coyne K, LoCasale R, Datto C, Sexton C, Yeomans $\mathrm{K}$, Tack J. Opioid-induced constipation in patients with chronic noncancer pain in the USA, Canada, Germany and the UK: descriptive analysis of baseline patient-reported outcomes and retrospective chart review. Clinicoecon Outcomes Res. 2014;6:269-81.

26. Poelaert J, Koopmans-Klein G, Dioh A, et al. Treatment with prolonged-release oxycodone/naloxone improves pain relief and opioid-induced constipation compared with prolonged-release oxycodone in patients with chronic severe pain and laxative-refractory constipation. Clin Ther. 2015;37:784-92. 